\title{
Possible Migration of an Antecubital Entry Sewing Needle to the Axillary Region: Case Report
}

\author{
Mehmet Burtac EREN* \\ Department of Orthopaedics and Traumatology, Gaziosmanpasa University School of Medicine, Tokat, Turkey
}

\begin{abstract}
Introduction: Many foreign body penetrations can occur in the limb. Common puncture injuries to the upper extremity involve items such as glass, metal, and wood splinters, plant thorns, and fish spines.

Case report: We report on the case of a foreign body that migrated in the early period after entry through the elbow antecubital fascia to the shoulder pectoralis major fascia.

A 20-year-old girl was seen in the emergency department after the penetration of a needle into her elbow region.

Puncture detected in the antecubital region. X-rays has showed metal needle in the axillary region. Needle was removed from the pectoralis major fascia.

Discussion: Our study, which can be seen as the first publication to report migration through the forearm and arm fascia, highlights the importance of penetrating needle injuries in the anterior elbow to prevent possible lethal complications and morbidity.
\end{abstract}

\section{Keywords}

Needle migration, Forearm fascia, Pectoralis major fascia

\section{Introduction}

Many foreign body penetrations can occur in the limb. Penetration can occur with different material types. Metal needles penetration especially related to sewing needles, is common [1].

Common puncture injuries to the upper extremity involve items such as sewing neddle, glass, metal, wood splinters, plant thorns, and fish spines. Most often, these injuries are resolved with little or no medical intervention. Some common complications from retained foreign bodies include persistent pain, infection, and tendon or nerve damage [2]. Migration of a foreign body in the body from a distal to proximal location has been reported in a limited number of cases [1-5]. Only one of these studies described penetration to forearm and migration at a chronic stage [3].

In this article, we have reported the case of a foreign body that migrated in the early period after entry through the antecubital region to the shoulder pectoralis major fascia. Foreign body migration should be kept in mind when evaluating patients that have penetrating injury to antecubital region.

\section{Case Report}

A 20-year-old girl was seen in the emergency department after the penetration of a needle from her clothing into her elbow region; this had occurred one day prior.
When she looked at elbow anterior surface, she saw a puncture in the antecubital region. The patient, who did not describe pain after the injury, applied to the emergency room of our hospital due to the presence of pain around the shoulder one day later. She was initially seen by a general practitioner and radiographs of the elbow were performed. After radiographs were taken, aconsultation was requested by us. The puncture in the antecubital region was documented, but no foreign body was visualized on the elbow radiographs (Figure 1).

Although the entry site was on the anterior side, the elbow joint movements were complete and painless, while shoulder movements and pain in the anterior part of the shoulder were observed. When a shoulder X-ray was taken due to the patient's pain in the shoulder region, the metal needle was observed to be in the axillary region (Figure 2).

*Corresponding author: Mehmet Burtac Eren, Department of Orthopaedics and Traumatology, Gaziosmanpasa University, Kaleardı District Muhittin Fisunoglu Street, 60100 Tokat, Turkey, Tel: +90-356212-9500-1282, GSM: +90532-483-6612

Accepted: October 08, 2020

Published online: October 10, 2020

Citation: EREN MB (2020) Possible Migration of an Antecubital Entry Sewing Needle to the Axillary Region: Case Report. J Orthop Surg Tech 3(2):175-178 


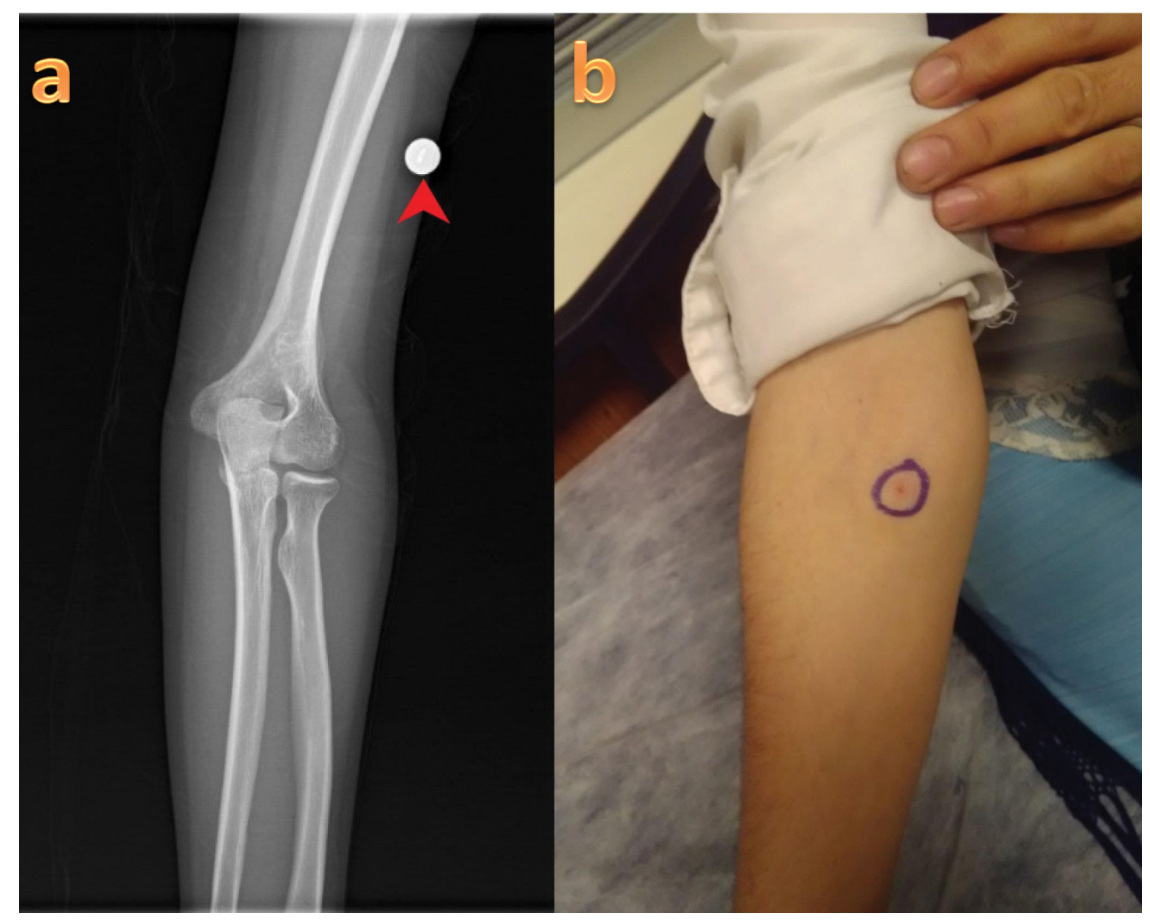

Figure 1: (a) Elbow radiography of the patient (Red arrow: Coin placed for side mark); (b) Clinical view of the needle insertion site in the antecubital region.

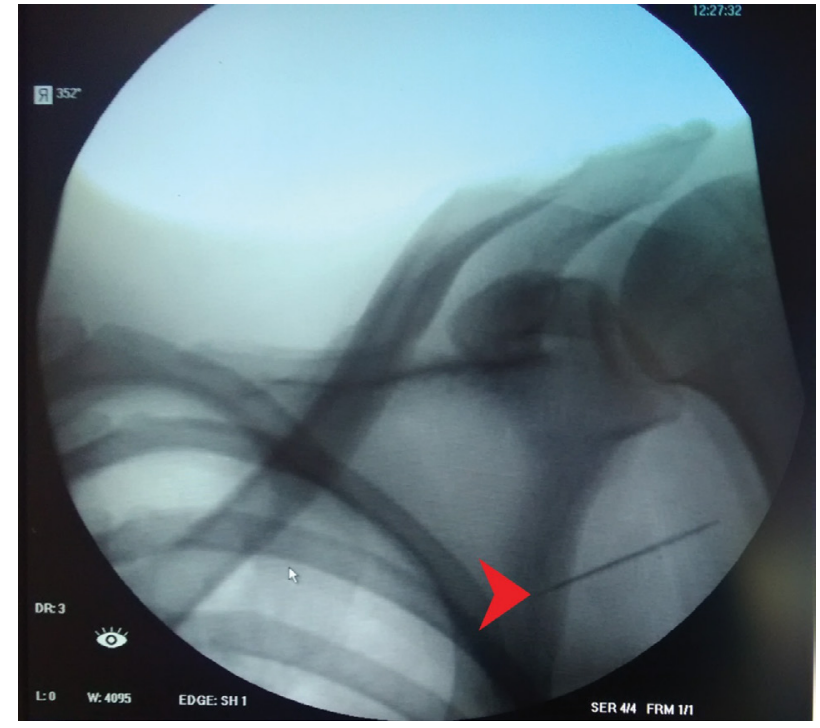

Figure 2: Fluoroscopic image of the needle (red arrow).

Her white blood cell count, erythrocyte sedimentation rate, and C-reactive protein levels were normal. Computed tomography of shoulder was requested in order to fully understand the location of the foreign body in the axillary region. Tip of coracoid process and tuberculum minus was accepted as a reference point in the $\mathrm{CT}$ examination. The distance of the needle to the coracoid process and tuberculum minus was calculated with surgical ruler, and its extension and placement were calculated (Figure 3).

Following palpation, the needle was removed from the pectoralis major fascia (Figure 4).

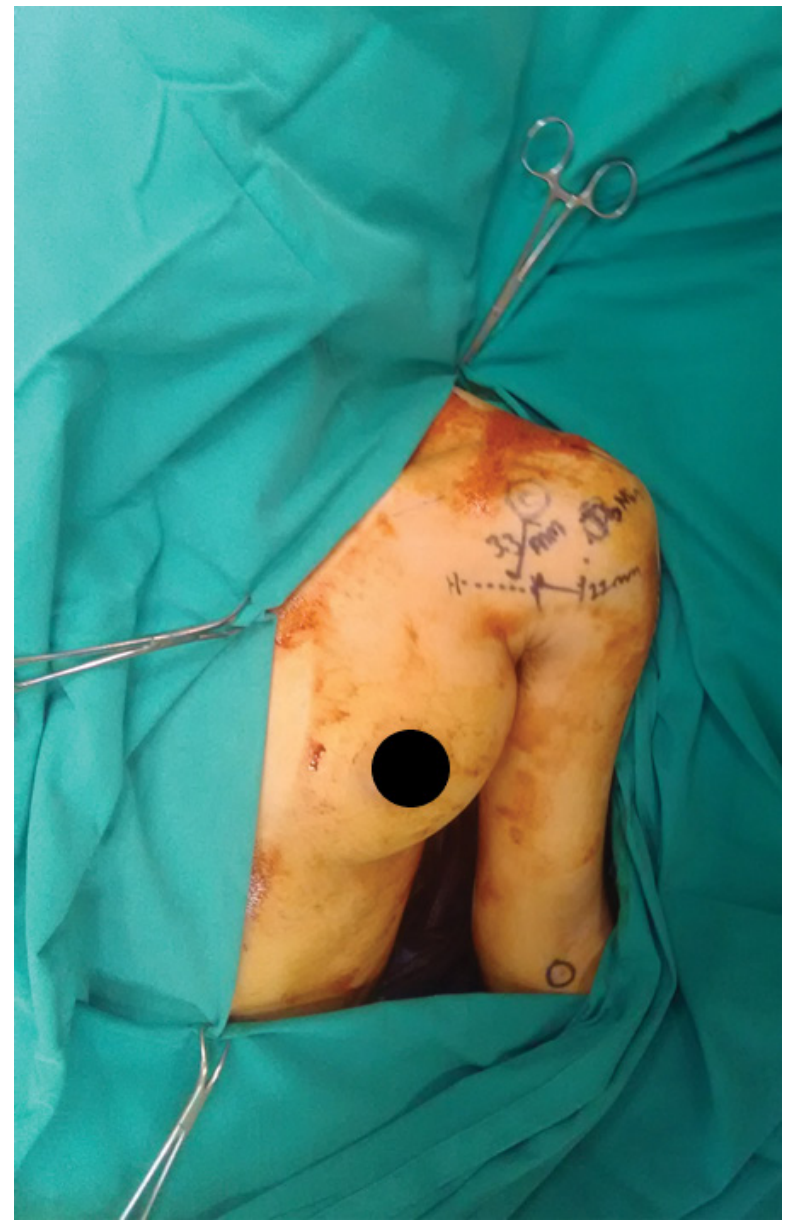

Figure 3: Planning the incision (dotted line) according to reference points. 


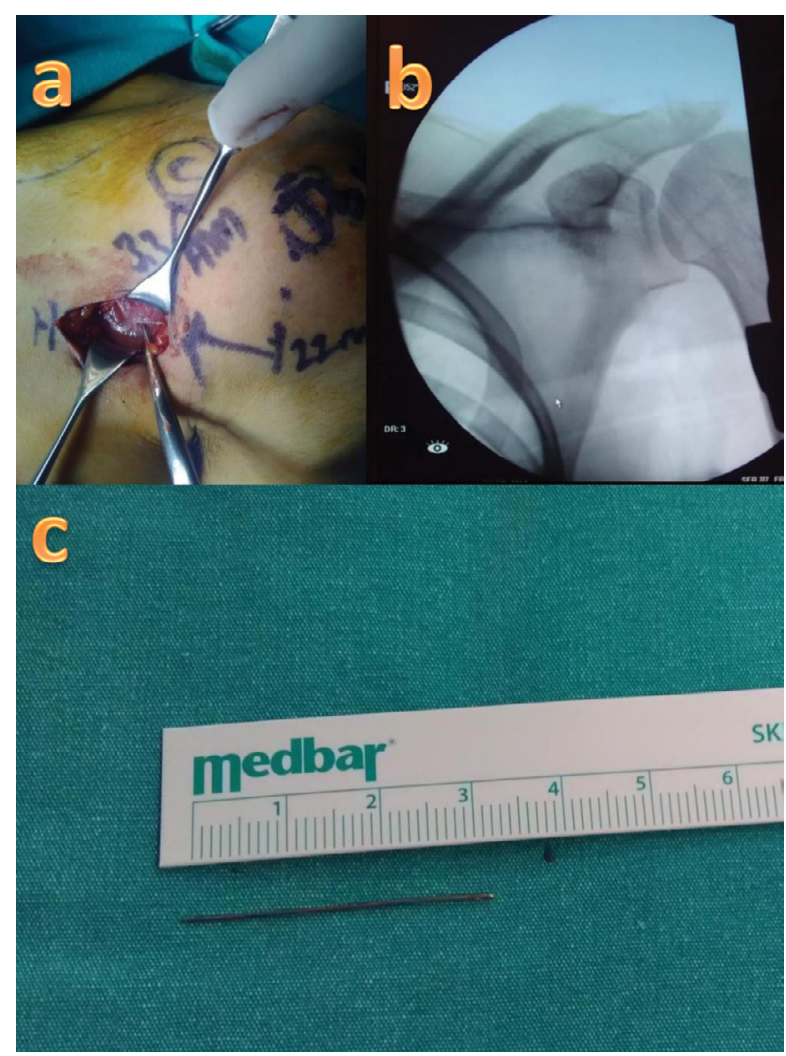

Figure 4: (a) View of the needle on the pectoralis major fascia; (b) Radiological appearance after needle removal; (c) Close view of the needle.

The distance between the entry point of the needle and the area from which it was removed was measured $22 \mathrm{~cm}$ with ruler. The patient was discharged from the hospital one day after the operation, and the wound healed without complication.

\section{Discussion}

Distal and proximal migration has been reported, especially along the tendon sheath in the upper extremity. Hand is one of the most common areas of foreign body penetration [6]. In a retrospective study, analysis of 200 patients who were evaluated for foreign body in the hand showedthat $38 \%$ of patients admitted due to foreign body reported a lack of diagnosis [2]. Choudhari, et al. [3] reported that the foreign body migration around the ulnar nerve in a patient who had been operated on due to ulnar neuropathy 18 months after foreign body penetration to the forearm region.

Seromas are known to occur frequently due to a potentially resistant dead space in the axillary region after axillary lymph node dissection [7-11]. Stanczyk, et al. [12] reported a seroma case associated with the pectoralis major fascia after radical mastectomy. In our case, the fact that the foreign body was located on the pectoralis major fascia may be important in terms of showing that foreign bodies in the arm can move towards the axillary area.

In our case, it seems quite interesting that the migration was about $22 \mathrm{~cm}$ in a day. This case, which probably occurred along the forearm fascia, looks quite different from the exam- ples in the literature because it occurred earlier and spread across the arm fascia. The patient cannot accurately describe the penetration direction of the sewing needle left on his suit. A vertical penetration is less likely to later become parallel to the skin. Therefore, needle penetration probably occurred parallel to the skin. There may be a rapid progression through the fascia with the contractions of the muscles around the antecubital region and arm. It is known that rapid migrations reported in the literature generally occur as a result of vascular transport [13-16]. It is known that migrations that occur along the tendon sheath occur relatively slowly $[5,6,17]$. Since the migration that occurred in our case probably occurred through the fascia, it seems more likely that the progression occurred rapidly with the contractions of the muscles.

Considering the continuity of the pectoralis major superficial fascia with the deep cervical fascia, there may be a risk of progression and injury to the cervical region [18]. Therefore, if our case had not obtained care early, she might have arrived with a different clinical scenario.

Since the foreign body in our case was metal, direct X-ray and tomography tests were sufficient in the evaluation. It may be necessary to use ultrasonography and MRI, especially with penetrations of woody material such as toothpicks $[17,19]$. Injuries with potential mortality have also been reported, such as needles advancing toward the cardiac area or those that have led to peritonitis after vascular access $[13,14]$. There are also publications reporting that acupuncture needles have migrated up to the thoracic cavity, heart, lung, and even the medulla oblongata $[15,16,20]$.

Our case presentation, which can be seen as the first publication reporting migration from the forearm and arm fascia, drew attention to the potential for foreign body migration in this region.

\section{Conflict of Interest}

The authors declare that they have no conflict of interest.

\section{Funding}

There is no funding source.

\section{Ethical Approval}

This article does not contain any studies involving animals performed by any of the authors. All procedures performed in studies involving human participant were in accordance with the ethical standards of the institutional and/or national research committee and with the 1964 Helsinki Declaration and its later amendments or comparable ethical standards.

Informed consent was obtained from individual.

\section{References}

1. Merrell JC, RC Russell, EG Zook (1983) Nonsuppurative tenosynovitis secondary to foreign body migration. Journal of Hand Surgery 8: 340-341.

2. Anderson MA, WL Newmeyer III, ES Kilgore Jr (1982) Diagnosis and treatment of retained foreign bodies in the hand. The American Journal of Surgery 144: 63-67. 
3. Choudhari K, T Muthu, M Tan (2001) Progressive ulnar neuropathy caused by delayed migration of a foreign body. British Journal of Neurosurgery 15: 263-265.

4. Chow J, RR Schenck (1988) Foreign body migration in the hand. Journal of Hand Surgery 13: 462.

5. Yang S, B Bear, A Weiland (1995) Rupture of the flexor pollicis longus tendon after 30 years due to migration of a retained foreign body. The Journal of Hand Surgery: British \& European 20: 803-805.

6. Jozsa L, A Reffy, Susanne Demel, et al. (1989) Foreign bodies in tendons. Journal of Hand Surgery 14: 84-85.

7. Gonzalez EA, Edward C Saltzstein, Carola S Riedner, et al. (2003) Seroma formation following breast cancer surgery. The Breast Journal 9: 385-388.

8. Tejler G, K Aspegren (1985) Complications and hospital stay after surgery for breast cancer: A prospective study of 385 patients. British Journal of Surgery 72: 542-544.

9. Esmat Hashemi, Ahmad Kaviani, Masoume Najafi, et al. (2004) Seroma formation after surgery for breast cancer. World Journal of Surgical Oncology 2: 44.

10. Woodworth PA, MF McBoyle, SD Helmer, et al. (2000) Seroma formation after breast cancer surgery: Incidence and predicting factors/discussions. The American Surgeon 66: 444-450.

11. Gambardella C, Guglielmo Clarizia, Renato Patrone, et al. (2019) Advanced hemostasis in axillary lymph node dissection for locally advanced breast cancer: New technology devices compared in the prevention of seroma formation. BMC Surgery 18: 1-9.
12. Stanczyk M, Bartlomiej Grala, Tomasz Zwierowicz, et al. (2007) Surgical resection for persistent seroma, following modified radical mastectomy. World Journal of Surgical Oncology 5: 1-4.

13. Ono M, RK Wolf (2001) Heart injury and endocarditis caused by a needle migrated from the left arm. European Journal of Cardio-Thoracic Surgery 20: 1074-1074.

14. Yu JC, KK Cheng (1975) Migration of broken sewing needle from left arm to heart. Chest 67: 626-627.

15. Hasegawa J, N Noguchi, J Yamasaki, et al. (1991) Delayed cardiac tamponade and hemothorax induced by an acupuncture needle. Cardiology 78: 58-63.

16. Neely D, R Jeganathan, G Campalani (2010) Transcaval migration of an acupuncture needle from the abdominal cavity to the heart. Journal of Cardiac Surgery 25: 654-656.

17. Albay C, Oktay Adanır, Sever Çağlar, et al. (2016) Migration of a toothpick along the flexor tendon sheath in a lower extremity. Acta Orthop Traumatol Turc 50: 245-247.

18. Stecco A, StefanoMasiero, VeronicaMacchi, et al. (2009) The pectoral fascia: Anatomical and histological study. Journal of Bodywork and Movement Therapies 13: 255-261.

19. Monu J, CM McManus, WG Ward, et al. (1995) Soft-tissue masses caused by long-standing foreign bodies in the extremities: MR imaging findings. AJR. American Journal of Roentgenology 165: 395-397.

20. Abumi K, H Anbo, K Kaneda (1996) Migration of an acupuncture needle into the medulla oblongata. European Spine Journal 5: 137-139. 